\title{
Effects of L-2-Oxothiazolidine-4-carboxylate on isoproterenol-induced acute myocardial infarction in rats
}

\author{
Marija Angelovski*, Dino Atanasov, Mitko Mladenov, Nikola Hadzi-Petrushev \\ Faculty of Natural Sciences and Mathematics, Arhimedova 3, 1000 Skopje, Republic of North Macedonia
}

\section{Introduction}

Myocardial ischemia occurs when myocardial oxygen demand exceeds oxygen supply leading to myocardial infarction (MI), which is one of the most lethal manifestations of cardiovascular diseases. Reactive oxygen species (ROS) and the associated oxidative stress are contributors to the pathological changes following MI (Lefer and Grabger, 2000). Isoproterenol (ISO), a synthetic catecholamine and beta-adrenergic agonist, induces oxidative stress in the myocardium, which may be a causative factor for irreversible damage of the myocardial membrane (Suchalatha and Shyamala, 2004). To prevent the damage by ROS, various antioxidant defense systems are present in the myocardial tissue and the glutathione/glutathione peroxidase system appears to be the main and the most active mechanism (Singh et al., 1989). The depletion of the antioxidant mechanisms renders cells particularly vulnerable to oxidative stress. Hence, the enhancement of the myocardial glutathione system might have protective effects.

Considering the limited effectiveness of the direct administration of glutathione, and the advantages of L-2-Oxothiazolidine-4-carboxylate (OTC) in enhancing the glutathione levels compared to other prodrugs (Cacciatore et al., 2010), this study examined the beneficial effects of OTC supplementation during the development of MI, by evaluating the cardiac glutathione content, the markers for cardiac lipid and protein oxidation, and the histopathology of the myocardium.

\section{Materials and methods}

\section{Animals and experimental design}

Male Wistar rats (200-250 g BW, $\mathrm{n}=32$ ) were divided into four equal groups. The rats in the OTC and in the OTC+ISO groups were treated with OTC (6.5 mmol/kg BW, i.p.) twice a day for two consecutive days. Rats from the groups ISO and OTC+ISO were submitted to acute administration of ISO (100 mg/kg BW, s.c.), two injections separated by an interval of $24 \mathrm{~h}$ (that is one ISO injection between the two daily OTC treatments for the OTC+ISO group). The rats in the control group (C) have received vehicles only. Twenty-four hours after the last ISO injection, the animals were sacrificed and heart samples were collected.

Assays for MDA, AOPP and total glutathione (GSH) in the heart and standard microscopy

Tissue assays were performed according to the method described by Ohkawa et al. (1979) and as described by Taylor et al. (2015) for MDA and AOPP, respectively. The enzymatic recycling method was employed for the determination of glutathione in the heart tissue (Rahman et al., 2006). Thin longitudinal ventricular sections were made and then stained with hematoxylin and eosin (H\&E) to detect leukocytes infiltration, while Masson's trichrome staining was used to spot myocardial necrosis.

\footnotetext{
*marija_bogdanovska@yahoo.com
} 


\section{Statistical Analysis}

Groups of data were compared using one-way analysis of variance. The post-hoc test of Tukey was performed in selected instances to evaluate further differences between group pairs.

\section{Results and discussion}

ISO administration in absence of OTC treatment (ISO group) caused significant increase in the levels of heart MDA and AOPP ( $p<0.01$ in both cases), and a significant decrease in the level of GSH $(p<0.05)$ compared to the control rats. These changes in the oxidative stress markers may be related to the ISO-induced increased ROS generation (Garg and Khanna, 2014), accompanied by insufficient antioxidant capacity in the myocardium. Indeed, decreased GSH levels have been observed in ISO-induced rats due to the increased utilization of protective thiol containing proteins by the lipid peroxides (Nagoor Meeran and Mainzen Prince, 2011). In our study, the treatment with OTC did not increase the myocardial levels of GSH in normal rats (OTC group compared to the controls), and did not prevent the ISO-associated increase in AOPP ( $\mathrm{p}<0.001$ for $\mathrm{C} v s$. OTC+ISO). However, the rats in the OTC+ISO group had cardiac GSH levels that were not significantly different compared to the controls. More importantly, the OTC treatment successfully reduced the ISO-associated increase in MDA (both comparisons "OTC+ISO vs. C" and "OTC+ISO vs. OTC" were ns). The obtained results suggest that OTC may have the ability to inhibit the deleterious effects induced by free radicals in ISOinjected rats, by means of GSH recovery that aids the maintenance of the redox balance in the cardiomyocytes (Tavares et al., 2012) and reduces the severity of the lipid peroxidation. This notion was also supported by the histological findings in the study. The myocardium of ISO-injected rats showed infarcted zone with large areas of necrosis, inflammatory cells, and separation of muscle fibers. OTC treatment reduced these histopathological changes. Additionally, the biochemical and the histopathological findings for the OTC group indicated that the OTC treatment, applied in large and frequent doses, does not possess any adverse effects.

\section{Conclusion}

This study showed that OTC mitigates ISOinduced cardiac changes. OTC treatment helps the maintenance of cardiac GSH levels, enabling the endogenous antioxidant mechanisms to cope more successfully with the ROS-induced lipid peroxidation.

\section{References}

Cacciatore, I., Cornacchia, C., Pinnen, F., Mollica, A., Di Stefano, A., 2010. Prodrug approach for increasing cellular glutathione levels. Molecules 15, 12421264.

Garg, M., Khanna, D., 2014. Exploration of pharmacological interventions to prevent isoproterenol-induced myocardial infarction in experimental models. Ther. Adv. Cardiovasc. Dis. 8, 155-169.

Lefer, D.J., Grabger, D.N., 2000. Oxidative stress and cardiac disease. Am. J. Med. 109, 315-323.

Nagoor Meeran, M.F., Mainzen Prince, P.S., 2011. Protective effects of $\mathrm{N}$-acetyl cysteine on lipid peroxide metabolism on isoproterenol-induced myocardial infarcted rats. J. Biochem. Mol. Toxicol. $25,151-157$.

Ohkawa, H., Ohishi, N., Yagi, K., 1979. Assay for lipid peroxides in animal tissues by thiobarbituric acid reaction. Anal. Biochem. 95, 351-358.

Rahman, I., Kode, A., Biswas, S.K., 2006. Assay for quantitative determination of glutathione and glutathione disulfide levels using enzymatic recycling method. Nat. Protoc. 1, 3159-3165.

Singh, A., Lee, K.J., Lee, C.Y., Goldfarb, R.D., Tsan, M.F., 1989. Relation between myocardial glutathione content and extent of ischemiareperfusion injury. Circulation 80, 1795-1804.

Suchalatha, S., Shyamala Devi, C.S., 2004. Effect of arogh - a polyherbal formulation on the marker enzymes in isoproterenol induced myocardial injury. Ind. J. Clin. Biochem. 19, 184-189.

Tavares, A.M., da Rosa Araujo, A.S., Llesuy, S., Khaper, N., Rohde, L.E., Clausell, N., Belló-Klein, A., 2012. Early loss of cardiac function in acute myocardial infarction is associated with redox imbalance. Exp. Clin. Cardiol. 17, 263-267.

Taylor, E.L., Armstrong, K.R., Perrett, D., Hattersley, A.T., Winyard, P.G., 2015. Optimisation of an advanced oxidation protein products assay: its application to studies of oxidative stress in diabetes mellitus. Oxid. Med. Cell. Longev. Available at: https://doi.org/10.1155/2015/496271. 